\title{
RO-VIBRATIONAL STATE DENSITIES BASED ON SPECTROSCOPIC DATA FOR NON-SEPARABLE SYSTEMS
}

\author{
Beatriz M. TOSELLI and John R. BARKER ${ }^{1}$ \\ Department of Atmospheric, Oceanic and Spaces Sciences, Space Physics Research Laboratory. The University of Michigan, \\ Ann Arbor, MI 48109-2143, USA
}

Received 20 April 1989; in final form 8 May 1989

\begin{abstract}
A Monte Carlo procedure for calculating the density of states is described. The method is based on an expansion of the eigenstate energies as term values, and coupling among vibrations and rotations is explicitly included. The accuracy of the technique for state densities is demonstrated by comparisons with "exact" results obtained form the differentiated sum of states. The applicability of the method is general, when reliable high order spectroscopic data are available. Calculations are presented for $\mathrm{NO}_{2}$, $\mathrm{H}_{2} \mathrm{O}, \mathrm{HOCl}$ and $\mathrm{CH}_{2} \mathrm{O}$ for energies $\geqslant 20000 \mathrm{~cm}^{-l}$.
\end{abstract}

\section{Introduction}

The calculation of the densities of vibrational and rotational energy levels and of the corresponding sum of states is important for numerous theoretical applications, including theories of unimolecular reactions, energy transfer, radiationless transitions, multiphoton absorption processes, and spectroscopic measures of molecular chaos. The current techniques available for the calculation of densities of states, $N(E)$, include direct count [1], semiempirical [2] and inverse Laplace transform [3] methods. The Beyer-Swinehart algorithm [4], which has very high efficiency, and its extension by Stein and Rabinovitch [5] enable the exact calculation of $N(E)$ for separable degrees of freedom. Indeed, inherent in most of these methods is the assumption that the system can be treated as a collection of independent (separable) oscillators. This is not because the couplings among the different degrees of freedom (DOF) are thought to be unimportant, but because they are difficult to evaluate. Methods for evaluating state densities that can incorporate the effects of non-separability include those based on classical mechanics and a recently described Monte Carlo method for sums of quantum states [6]; "exact" densities of

\footnotetext{
1 And Department of Chemistry.
}

states can be obtained by differentiating the sum of states with respect to energy.

In classical statistical mechanics the density of states at energy $E$ for a system with $n$ degrees of freedom is given by

$$
N(E)=h^{-s} \iint \mathrm{d} p \mathrm{~d} q \delta[E-H(p, q)],
$$

where the coordinates $q$ and conjugate momenta $p$ are the variables of the classical Hamiltonian $H(p$, $q)$, the Dirac delta function ensures the integration in eq. ( 1 ) is carried out for $H(p, q)=E$. Except for very simple models, such as the harmonic oscillator and rigid rotor, the integral (1) has no closed form solution, and for real systems serious approximations may be necessary for its evaluation. The numerical evaluation of eq. (1) has been attacked using Monte Carlo methods which are particularly suitable for multidimensional integration [7]. Several papers have shown the usefulness of the Monte Carlo techniques $[8,9]$, which do not depend on the particular degrees of freedom or their coupling.

In a recent paper [6], a high efficiency Monte Carlo integration technique was described that gives unbiased estimates and can be used to calculate the sum of quantum states for nonseparable degrees of freedom. This technique does not evaluate eq. (1), but it is based on spectroscopic term expressions for 
the eigenstate energies in terms of quantum numbers. The approach followed in the present paper is largely based on the method of ref. [6] and the reader should consult the original paper for a complete explanation of that method and an outline of the sampling algorithm. The density of states can be determined by taking the derivative of the sum of states with respect to energy. This necessitates the determination of the sum of states at several energies, fitting the results to an analytical function, and then taking the derivative. In the present work, a much more efficient approximate method is described, which retains all of the benefits of the Monte Carlo integration, but without the added labor, and which makes only a small sacrifice in accuracy.

\section{Formalism}

The energy of an eigenstate (relative to the zeropoint energy) for a polyatomic system, in the ground electronic state (for a symmetric top) can be written

$T=G^{0}\left(J, K, v_{i} \ldots \nu_{n}\right)+E_{\text {rot }}(J, K)$,

where the spectroscopic terms for anharmonic rovibrational eigenstates are represented empirically by an expression in powers of rotational $(J, K)$ and vibrational quantum numbers, $v_{i}$ (in the absence of resonances and other perturbations). The terms in eq. (2) are given by

$$
\begin{aligned}
G^{0} & =\sum_{i=1}^{n} v_{i} \omega_{i}^{0 \mathrm{eff}}+\sum_{i=1}^{n} \sum_{j=i}^{n} X_{i j}^{0 \mathrm{eff}} v_{i} v_{j} \\
& +\sum_{i=1}^{n} \sum_{j=i}^{n} \sum_{k=j}^{n} y_{i j k} v_{i} v_{j} v_{k},
\end{aligned}
$$

where $\omega_{i}^{0 \text { eff }}$ and the $X_{i j}^{0 \text { eff }}$ are the effective vibrational frequency and effective quadratic anharmonicities and $y_{i j k}$ are the cubic anharmonicities. Due to the coupling with rotations, the effective frequencies and quadratic anharmonicities are functions of the rotational quantum numbers

$$
\begin{aligned}
& \omega_{i}^{\text {Oeff }}(J, K)=\omega_{i}^{\mathrm{O}}-\alpha_{i}^{\mathrm{B}}\left[J(J+1)-K^{2}\right] \\
& \quad-\alpha_{i}^{\mathrm{A}} K^{2}+\beta_{i} K^{4}, \\
& X_{i i}^{\text {oeff }}(J, K)=X_{i j}^{0}+\gamma_{i i}^{\mathrm{A}} K^{2} .
\end{aligned}
$$

The rotational energy $E_{\mathrm{rot}}(J, K)$ (according to the
Watson Hamiltonian [10]) is given by

$$
\begin{aligned}
& E_{\mathrm{rot}}(J, K)=B_{0} J(J+1)+\left(A_{0}-B_{0}\right) K^{2} \\
& \quad-A_{J} J^{2}(J+1)^{2}-\Delta_{J K} J(J+1) K^{2}-\Delta_{K} K^{4} \\
& +H_{J} J^{3}(J+1)^{3}+H_{J K} J^{2}(J+1)^{2} K^{2} \\
& +H_{K J} J(J+1) K^{4}+H_{K} K^{6}-L_{K} K^{8} .
\end{aligned}
$$

The procedure we follow in calculating the density of rovibrational states is derived from that in ref. [6]. The basic idea is that, in a Monte Carlo trial, integer quantum numbers for all but one of the DOF are selected in such a way that a specified total energy $E$ is not exceeded. In the present work the quantum number for the remaining DOF is treated as a continuous variable and is assigned so that the total energy equals $E$. The use of the continuous variable makes possible the easy evaluation of the density of states for cach trial assignment of all of the other quantum numbers, as shown below. The total density of states is the sum of the densities of states obtained from every possible combination of quantum number assignments, which is obtained by a weighted Monte Carlo integration.

For convenience, we label each DOF and corresponding quantum number $v_{\alpha}$ with a subscript $i$, so that each DOF can be identified, regardless of order.

The procedure can be summarized as follows:

(1) For a total energy, $E$, and a system formed by $n$ DOF, we arbitrarily select one DOF $(i=x)$ to be treated classically (i.e. its quantum number is treated as a continuous variable). The quantum numbers $v_{i}$ corresponding to the other $n-1$ DOF are treated as discrete variables, but $v_{x}$ is treated as a continuous variable.

(2) For a given $J$, randomly select $K$, subject to the condition that $|K| \leqslant J$ and $E_{\mathrm{rot}}(J, K) \leqslant E$ (here, we assume $J$ is a good quantum number, but $K$ is not). With the assigned $J$ and $K$ values, calculate $\omega_{i}^{0 \text { eff }}$ and $X_{i j}^{0=e f}$ using eqs. (4) and (5).

(3) Randomly assign integer quantum numbers to the $n-1$ vibrational modes, subject to conservation of energy. The maximum value of $v_{i}$ for each DOF will depend on quantum numbers already selected for the other modes, due to the inter-mode couplings. In one dimension, $v_{\max }$ is the largest integer value of $v$ for which

$\partial E / \partial v_{i} \geqslant 0$. 
For multiple DOF, we must be sure that any combination of quantum numbers will produce states that are bound with respect to all DOF. Therefore, the condition ( 7 ) is generalized and the test is applied to all DOF, using the assigned quantum numbers:

$$
\left(\partial E / \partial v_{i}\right)_{v j \neq i}>0, \text { for } i=1 \text { to } n \text {. }
$$

(4) When integer quantum numbers have been assigned to all $n-1 \mathrm{DOF}$, except $v_{x}$, the energy available for $x$ can be written

$$
\begin{aligned}
& U\left(v_{x}\right)=E-\sum_{i=1}^{n-1} v_{i} \omega_{i}^{\text {oeff }}-\sum_{i=1}^{n} \sum_{j=i}^{n-1} X_{i j}^{\text {Oeff }} v_{j} v_{i} \\
& +\sum_{i=1}^{n} \sum_{j \geqslant i}^{n} \sum_{k \geqslant j}^{n-1} y_{i j k} v_{i} v_{j} v_{k}-E_{\mathrm{rot}}(J, K) \\
& =\left(\omega_{x}^{\text {oeff }}+\sum_{i=1}^{n-1} X_{i x}^{\text {neff }} v_{i}+\sum_{i=1}^{n-1} \sum_{j=i}^{n-1} y_{i j k} v_{i} v_{j}\right) v_{x} \\
& +\left(X_{x x}^{\text {Oeff }}+\sum_{i=1}^{n-1} y_{i j k} v_{i}\right) v_{x}^{2}+y_{x x x} v_{x}^{3} .
\end{aligned}
$$

Eq. (9) has the form $U\left(v_{x}\right)=\omega v_{x}+X v_{x}^{2}+y v_{x}^{3}$, from which $v_{x}$ (treated as a continuous variable) can be calculated (the tests given by eqs. ( 7 ) and ( 8 ) must also be performed for the last mode, where an integer $v_{x}$ can be estimated by rounding, or by truncation of the continuous value). For this trial, the density of bound states for $x$ th DOF in the field of all the other DOF is given by

$\rho_{x}(U)=\left[\left(\partial U / \partial v_{x}\right)_{v_{i+x}}\right]^{-1}$

Inspection of eqs. (9) and (10) shows that the effective one-dimensional term expression for the last DOF depends on all the other quantum numbers (due to the coupling).

(5) This procedure is repeated for many trials, according to the accuracy desired.

(6) The total density of bound states $N_{x}(E)$ obtained using the quantum number of $x$ th DOF as the continuous variable, is the weighted sum [6] of the contribution from each trial for a total of $S$ trials

$N_{x}(E)=\sum_{k=1}^{S} w_{k}\left[\rho_{x}(U)\right] k$

and the weighting factor for the $k$ th trial is calculated as $w_{k}=\prod_{m=1}^{n} R_{k m}^{-1}$,

where $R_{k m}$ is the range of the $m$ th variable in the $k$ th trial (see ref. [6] for discussion).

The density of states can be calculated in this manner with any DOF selected for classical treatment. Ideally, $N_{x}(E)$ should be independent of $x$ (which DOF is treated classically), but results shown below indicate that the approximation is most accurate when the lowest frequency DOF is treated in this manner.

\section{Results and discussion}

In order to test this approximate method, we have carried out calculations for several molecules including three triatomic systems: $\mathrm{NO}_{2}, \mathrm{H}_{2} \mathrm{O}$ and $\mathrm{HOCl}$ and one tetra-atomic: $\mathrm{H}_{2} \mathrm{CO}$. In each case, the "exact" density of bound states used in comparisons with the approximate results was found by differentiating the sum of states obtained using the method of ref. [6]. At very low energies and for molecules with high frequencies, the sums of states are very low and the corresponding densities are very sparse. Under these conditions, the density of states is not a well-defined continuous function and the whole concept breaks down. These are also the conditions that provide the most severe test for the approximate method.

Results for $\mathrm{NO}_{2}$ are presented in fig. 1. This molecule is particularly attractive because of the completeness of the spectroscopic data [11-13]. Results for $J=0$ and $J=10$ are presented in figs. $1 \mathrm{a}$ and $1 \mathrm{~b}$, respectively. We have not attempted calculations for higher values of $J$ because eq. (6) diverges for large values of the rotational quantum numbers. This is a common problem with spectroscopic data, because they were obtained at relatively low energies and extrapolations of the power-law expansions above the values for which they were fitted can produce nonsense energy values. In both panels the density of states obtained with the approximation is compared with the exact density of states, $N(E)_{\text {exact }}$, determined by fitting the exact sum of states from Monte Carlo method of ref. [6] to a polynomial and then taking the derivative. The agreement between the two methods is very good; the error of the approximate 

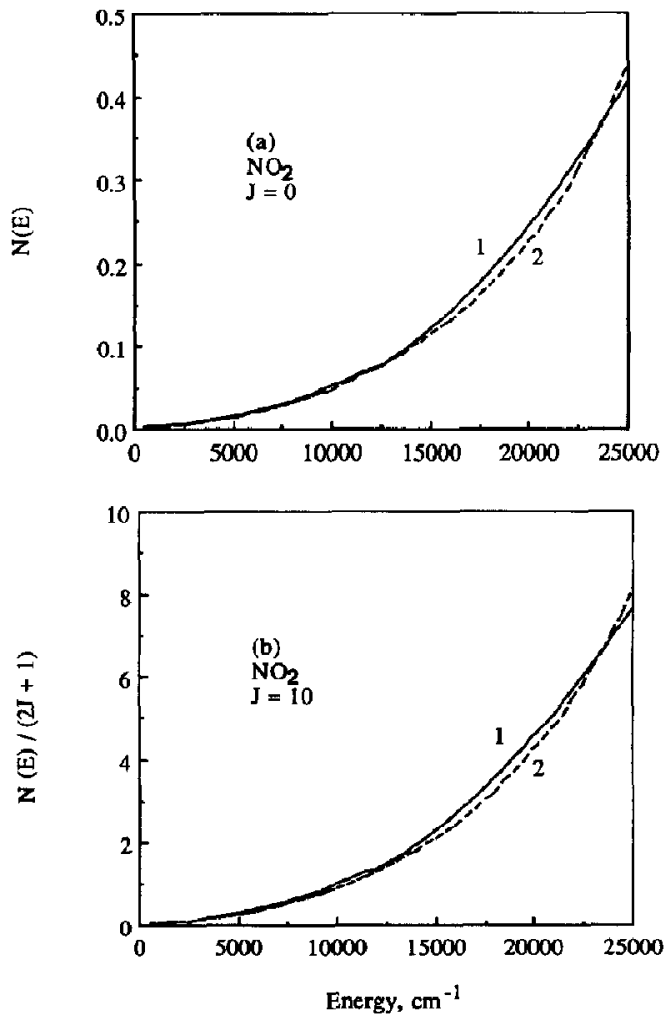

Fig. 1. Density of rovibrational states for $\mathrm{NO}_{2}$. (1) "Exact" density of states; (2) approximate method. Panel (a) $J=0$; panel (b) $J=10$.

method is less than $10 \%$ for energies up to the dissociation energy. The three vibrational frequencies in $\mathrm{NO}_{2}$ are similar and results obtained using each as $v_{x}$ are virtually identical.

Water provides an interesting test, because it has two high frequency modes $\left(\approx 3750 \mathrm{~cm}^{-1}\right)$ and a bending mode of moderate frequency $\left(1612 \mathrm{~cm}^{-1}\right)$, and so we can investigate the effect of $v_{x}$. For this molecule, the spectroscopic data were taken from a theoretical calculation [14] that reports the complete set of quadratic and cubic anharmonicitics. Moreover, the density of states is very low, providing a severe test of the approximate method. In fig. $2, N_{x}(E)$ for $x=1,2$ is compared with $N(E)_{\text {exact }}$, and it is clear from the figure that $x=2$ gives the better results. (The results obtained for $x=3$ are virtually identical to those for $x=1$ and are not shown.) The

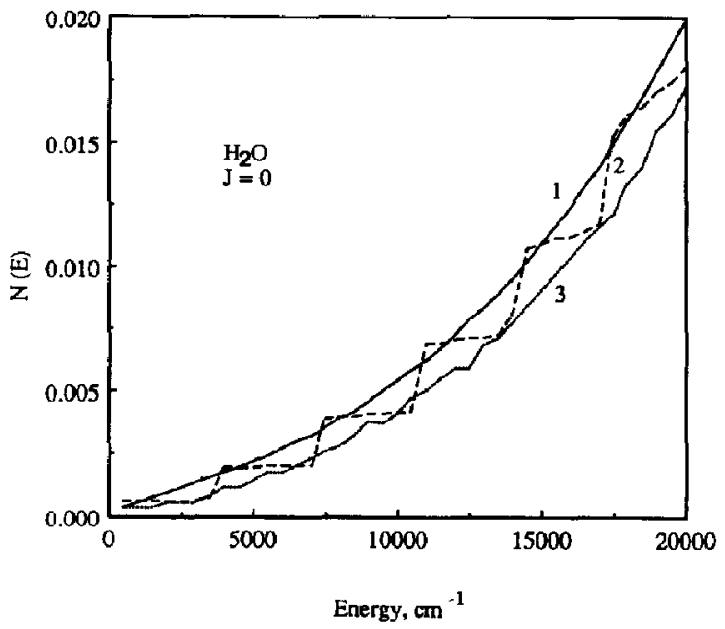

Fig. 2. Density of rovibrational states for $\mathrm{H}_{2} \mathrm{O}$. (1) "Exact" density of states; (2) approximate method, based on $\nu_{2}$; (3) approximate method, based on $\nu_{I}$.

steps apparent in the figure arise because the states are so sparsely distributed.

Note that $x=2$ is the lowest frequency mode in $\mathrm{H}_{2} \mathrm{O}$ and it gives the best estimate for the density of states. To determine whether this is a general tendency we carried out calculations for HOCl [15]. This molecule has mode frequencies that span a factor of five: $3811.93,1269.18$ and $741.7 \mathrm{~cm}^{-1}$. The results for each mode are shown in fig. 3 , and it is again clear that the best choice is the lowest frequency mode. For $N(E) \geqslant 0.02 / \mathrm{cm}^{-1}$, the approximate method gives results within about $10 \%$ of the exact values.

In fig. 4a, similar calculations are presented for $\mathrm{CH}_{2} \mathrm{O}$ [16], which has a density of states much higher than for the triatomic systems. In the inset, the ratio $N(E) / N(E)_{\text {exact }}$ is plotted for the lowest and the highest frequency modes as a function of the density of states. These results show that the lowest frequency mode is the best choice for application of the approximate method.

The fact that the lowest frequency mode provides the best estimate can be rationalized by noting that the technique is based on the reduction of a multidimensional problem to one dimension, and the corresponding quantum number is then treated as a continuous variable, rather than as an integcr. The lower the frequency, the better the continuous ap- 


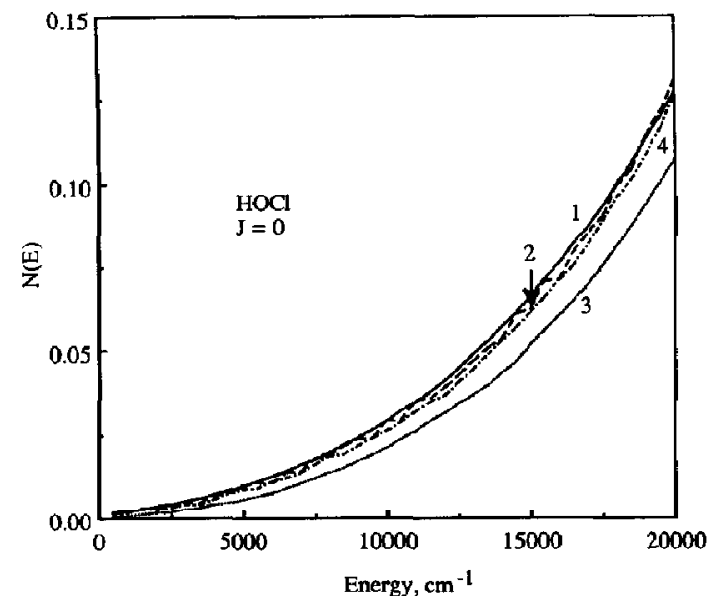

Fig. 3. Density of rovibrational states for HOCl. (1) "Exact" density of states; (2) approximate method, based on $\nu_{3}$; (3) approximate method, based on $\nu_{1}$; (4) approximate method, based on $v_{2}$.

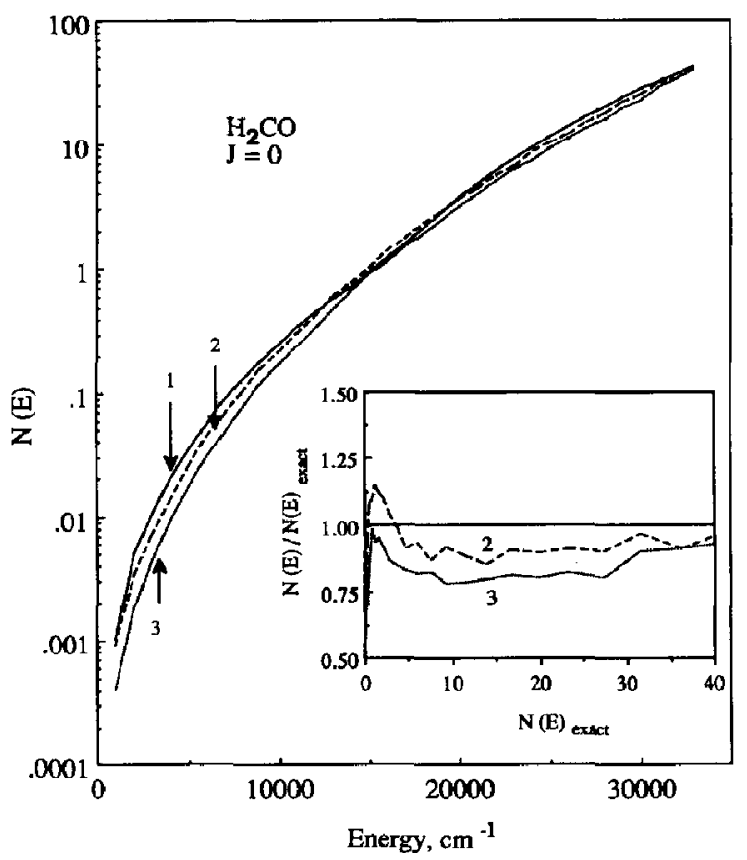

Fig. 4. Density of rovibrational states for $\mathrm{H}_{2} \mathrm{CO}$. (1) "Exact" density of states; (2) approximate method, based on lowest frequency mode; (3) approximate method, based on highest frequency mode. proximation. The fact that the lowest frequency mode provides the best estimate results in a significant saving of computational effort, specially for large molecules, because only one Monte Carlo calculation need be performed at each energy. Note that all the calculations reported here were performed with a Macintosh-II microcomputer and around $10^{4}$ trials produced results with statistical errors $\leqslant 10 \%$.

Extension of the methods described here and in ref. [6] to high values of $v_{i}, J$, and $K$ are straightforward in principle, since these Monte Carlo methods can accommodate all types of coupling. However, expressions such as eq. (6) are not always wellbehaved for some molecules and may become oscillatory and divergent for high values of the quantum numbers, as discussed by several authors (for example, see ref. [17]). It is beyond the scope of the present Letter to consider such problems, but practical solutions to this problem are needed for energies near and above reaction threshold energies, where the sums and densities of states are used to calculate unimolecular rate constants [18].

\section{Conclusions}

An efficient method for accurately calculating the density of states for coupled systems is presented. The method can be applied to any type of coupling for which an expression of the form of eq. (2) is available. The method should be useful in theoretical applications where the densities of states for coupled systems are needed. The results show the approximate method is accurate to within $\approx 10 \%$ for densities of states greater than $\approx 0.02 / \mathrm{cm}^{-1}$.

\section{Acknowledgement}

We thank the US Department of Energy, Office of Basic Energy Sciences for partial support of this research and BMT thanks CONICET of Argentina for a postdoctoral fellowship. 


\section{References}

[1] P.J. Robinson and K.A. Holbrook, Unimolecular reactions (Wiley-Interscience, New York, 1972).

[2] G.Z. Whitten and B.S. Rabinovitch, J. Chem. Phys. 38 (1963) 2466.

[3] W. Forst, Theory of unimolecular reactions (Academic Press, New York, 1973).

[4] T. Beyer and D.F. Swinehart, Commun. ACM 16 (1973) 379.

[5] S.E. Stein and B.S. Rabinovitch, J. Chem. Phys. 58 (1973) 2438.

[6] J.R. Barker, J. Phys. Chem. 91 (1987) 3849.

[7] J.M. Hammersley and D.C. Handscomb, Monte Carlo methods (Chapman and Hall, London, 1964).

[8] J.D. Doll, Chem. Phys. Letters 72 (1980) 139.

[9] S.C. Farantos, J.N. Murrell and J.C. Hajduk, Chem. Phys. 81 (1984) 828 .
[10] J.K.G. Watson, J. Chem. Phys, 46 (1967) 1935, 48 (1968) 4517.

[11] H.D. Bist and J.C.D. Brand, J. Mol. Spectry. 62 (1976) 60.

[12] W.J. Lafferty and R.L. Sams, J. Mol. Spectry. 66 (1977) 478.

[13] R.L. Sams and W.J. Lafferty, J. Mol. Spectry. 125 (1987) 99.

[14] G.A. Khachkuruzov, Opt. Spectry. 6 (1959) 294.

[15] L. Halonen and T.K. Ha, J. Chem. Phys. 88 (1988) 3775.

[16] D.E. Reisner, R.W. Field, J.L. Kinsey and H.L. Dai, J. Chem. Phys. 80 (1984) 5968.

[17] M.R. Aliev and J.K.G. Watson, in: Molecular spectroscopy: modern research, Vol. 3, ed. K.N. Rao (Academic Press, Orlando, 1985) p. 1.

[18] B.M. Toselli and J.R. Barker, J. Chem. Phys., in press. 